\title{
INCORRECT MATCH DETECTION METHOD FOR ARCTIC SEA-ICE RECONSTRUCTION USING UAV IMAGES
}

\author{
Jae-In Kim ${ }^{1}$, Hyun-cheol Kim ${ }^{1, *}$ \\ ${ }^{1}$ Unit of Arctic Sea-Ice Prediction, Korea Polar Research Institute (KOPRI), Incheon, Korea - (jikim0312, kimhc)@kopri.re.kr
}

\author{
Commission II, WG II/2
}

KEY WORDS: Unmanned Aerial Vehicle, Digital Surface Model, Image Matching, Arctic Sea-Ice

\begin{abstract}
:
Shapes and surface roughness, which are considered as key indicators in understanding Arctic sea-ice, can be measured from the digital surface model (DSM) of the target area. Unmanned aerial vehicle (UAV) flying at low altitudes enables theoretically accurate DSM generation. However, the characteristics of sea-ice with textureless surface and incessant motion make image matching difficult for DSM generation. In this paper, we propose a method for effectively detecting incorrect matches before correcting a sea-ice DSM derived from UAV images. The proposed method variably adjusts the size of search window to analyze the matching results of DSM generated and distinguishes incorrect matches. Experimental results showed that the sea-ice DSM produced large errors along the textureless surfaces, and that the incorrect matches could be effectively detected by the proposed method.
\end{abstract}

\section{INTRODUCTION}

The demand for Arctic sea-ice information is increasing rapidly for predicting Arctic climate change and for exploring northern sea route. Satellite-based remote sensing techniques can be effectively applied because Arctic sea-ice information production is based on a wide-area analysis. For developing satellite-based remote sensing techniques, field data are essential (Tschudi et al., 2008). However, in Arctic regions, it is not easy to obtain field data due to severe weather, inaccessible zones, and limited human resources. For these reasons, in the recent polar region researches, field data acquisition using unmanned aerial vehicles (UAVs) is being considered (Hagen et al., 2014; Divine et al., 2016). Although this acquisition method is limited in terms of the type and quality of the field data compared to the field survey by human investigators, it is possible to obtain more extensive and dense data. In addition, unlike high-resolution optical satellites, UAVs are free from cloudiness and are flexible in selecting data acquisition time.

In analysing Arctic sea-ice, shape and surface roughness are treated as key indicators. These can be measured from the digital surface model (DSM) for Arctic sea-ice. Therefore, acquisition of accurate DSM is important in developing satellite remote sensing techniques. Since UAVs can obtain high-resolution images through low altitude flight, it is also possible to generate high-quality DSMs with the UAV images. However, the characteristics of sea-ice, such as textureless surfaces and incessant motion, can cause serious errors in image matching process for DSM generation. In this case, the quality of the generated DSM is difficult to guarantee.

In this paper, we investigates a method for effectively detecting incorrect matches in Arctic sea-ice DSM. This is aimed at providing a way to increase efficiency in DSM correction. The proposed method focuses on textureless surface as the main cause of incorrect matches for sea-ice. This is due to the lack of texture information within the search window in terms of image matching (Veksler, 2003). For this reason, in our method, search window size is variably adjusted so that matching results can be reliably distinguished.

\section{INCORRECT MATCH DETECTION}

The proposed method assumes that interior and exterior parameters of cameras for image matching are given. Interior parameters include focal length, pixel size, principal point, and lens distortion coefficients of camera. Exterior parameters indicates position and attitude of camera. In the proposed method, incorrect matches are detected through matching image search, window size adjustment, and similarity measurement steps. This process is applied equally to all matches in a DSM generated. Figure 1 shows the workflow of the proposed method.

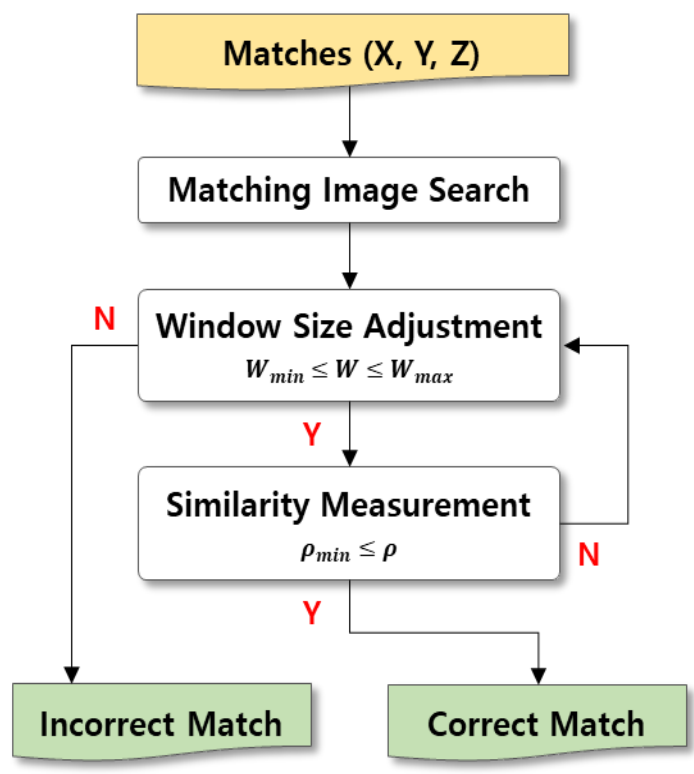

Figure 1. Workflow of the proposed method

In matching image search step, the images involved in the positioning of a given match are defined. This is based on the collinear condition that explains geometric relationship between ground point, image point, and perspective center. Figure 2

\footnotetext{
* Corresponding author
} 
illustrates the relationship between these three points. Collinear condition can be expressed as Equation 1.

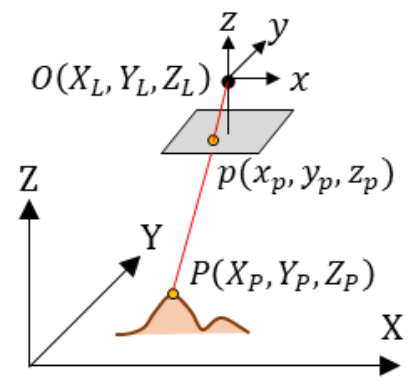

Figure 2. Geometric relationship between ground point, image point, and perspective center.

$$
\begin{aligned}
& x_{p}+\Delta x \\
& =-f \frac{r_{11}\left(X_{P}-X_{L}\right)+r_{12}\left(Y_{P}-Y_{L}\right)+r_{13}\left(Z_{P}-Z_{L}\right)}{r_{31}\left(X_{P}-X_{L}\right)+r_{32}\left(Y_{P}-Y_{L}\right)+r_{33}\left(Z_{P}-Z_{L}\right)} \\
& y_{p}+\Delta y \\
& =-f \frac{r_{21}\left(X_{P}-X_{L}\right)+r_{22}\left(Y_{P}-Y_{L}\right)+r_{23}\left(Z_{P}-Z_{L}\right)}{r_{31}\left(X_{P}-X_{L}\right)+r_{32}\left(Y_{P}-Y_{L}\right)+r_{33}\left(Z_{P}-Z_{L}\right)}
\end{aligned}
$$

where $\quad f=$ focal length

$r_{11}, \ldots, r_{33}=$ rotation matrix elements

$X_{P}, Y_{P}, Z_{P}=$ object coordinates of ground point $X_{L}, Y_{L}, Z_{L}=$ object coordinates of ground point $x_{p}, y_{p}=$ photo coordinates of ground point $\Delta x, \Delta y=$ offset of principal point

In window size adjustment step, size of search window for measuring similarity of matching images is determined. Window size should contain enough texture information to allow image matching to be successful. To this end, window size is gradually increased within a range of predefined minimum and maximum sizes. If window size exceeds the allowable value $\left(W_{\max }\right)$ during this process, the current match point is determined as an error.
In similarity measurement step, similarity between matching images is measured. This is accomplished by extracting patch images centered on a given match for each matching image, as in object-space-based image matching method (see Figure 2(a)). The representative value of similarity for the match is defined as the average of the similarity values measured between the patch images (see Fig. 2 (b)). If the average similarity is greater than the allowable value $\left(\rho_{\min }\right)$, the match is regarded as a matching success point. If the average similarity is less than the allowable value, the process returns to the window size adjustment step, and then the same processes are repeated.

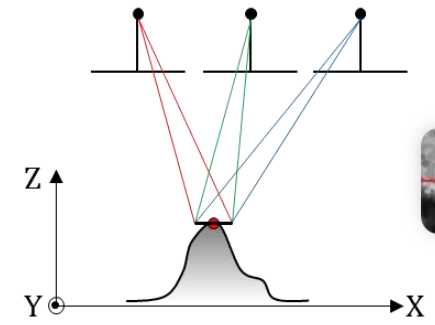

(a)

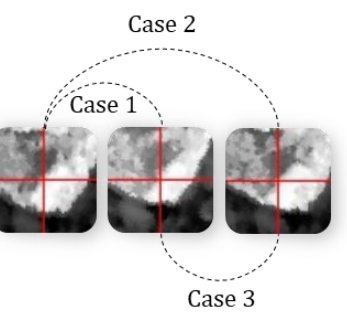

(b)
Figure 2. Similarity measurement. (a) patch image extraction and (b) similarity measurement.

\section{RESULT AND DISCUSSION}

We used twenty-five UAV images acquired at the Arctic sea-ice camp in August 2017. The camera mounted on the UAV was a DJI FC330 with an image size of $4000 \times 3000$, a focal length of $3.6 \mathrm{~mm}$, and a pixel size of $1.58 \mu \mathrm{m}$.

Since sea-ice is constantly moving over the sea, geo-referencing based on ground control points (GCPs) is practically impossible. Therefore, we produced a DSM using only GPS/INS information obtained with images. For generating the DSM, commercial software Pix4D (ver.4.1.24) was used. The spatial resolution of the DSM was $4.02 \mathrm{~cm}$. Figure 3 indicates the location of sea-ice camp and camera exposure points.
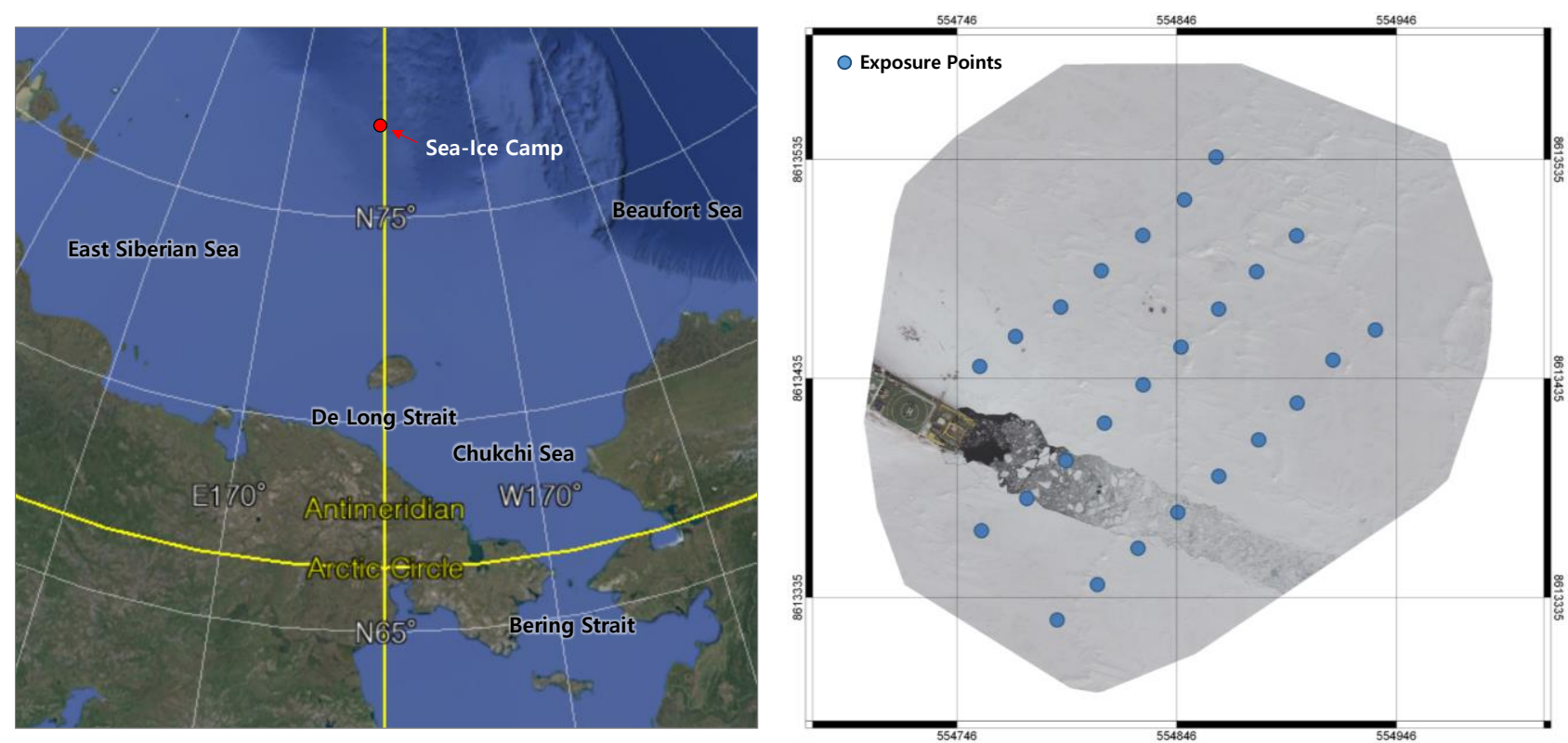

Figure 3. Sea-ice camp and camera exposure points for DSM generation. 


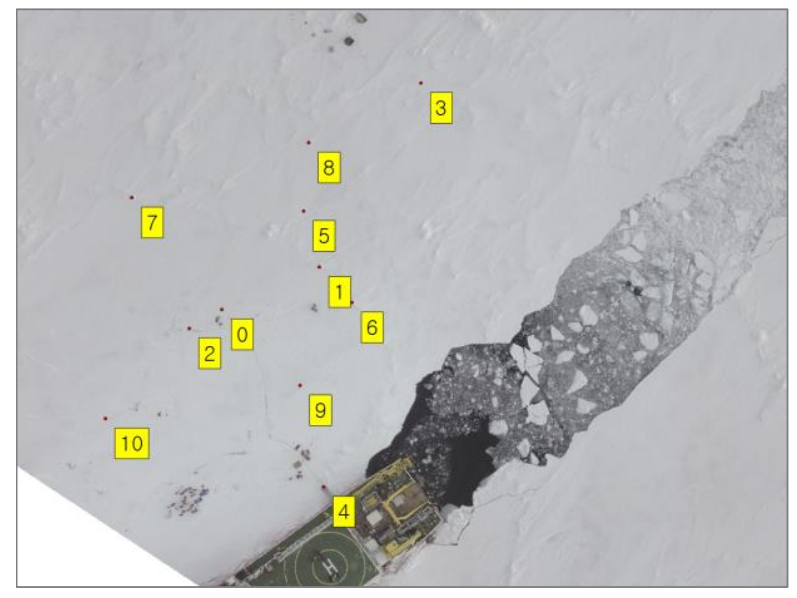

(a)

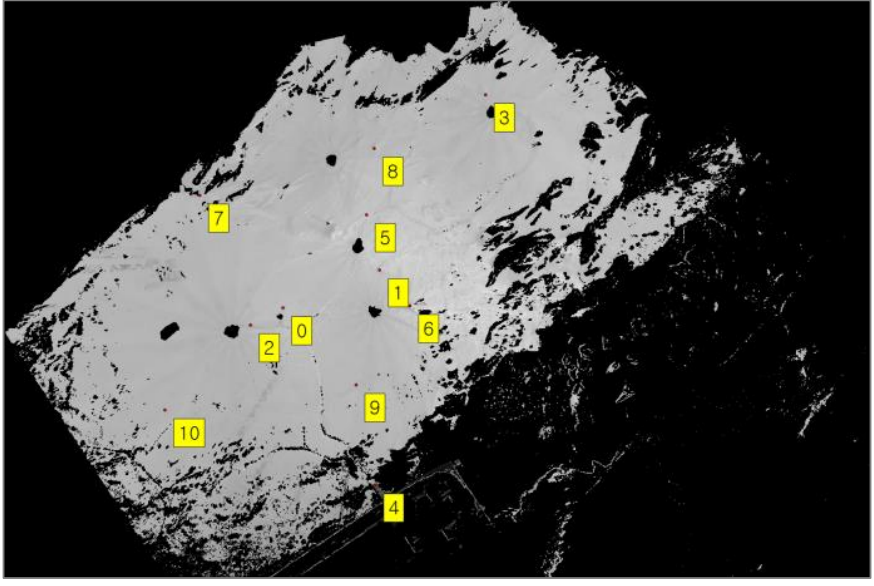

(b)

Figure 4. Tiepoints extracted for registration between the UAV DSM and the scanner DSM. (a) ortho-mosaic image for the UAV images and (b) ortho-mosaic image for the scanning data.

In order to evaluate the proposed method, colored point cloud was obtained by laser scanner, Trimble Focus 3D X130. Orthomosaic image and DSM were generated from the point cloud. Registration based on 3D conformal transformation was performed to match the coordinate systems between the two DSMs derived from the UAV images and the laser scanning data. Eleven 3D tiepoints for registration were manually extracted. The height values of tiepoints extracted from the UAV data were corrected using object space based matching method to consider the error of the UAV DSM. Figure 4 shows the tiepoints extracted from the two ortho-mosaic images, respectively.

Quality assessment of the UAV DSM was performed by comparing with the scanner DSM, which was done for the overlapping area between the UAV DSM and the scanner DSM as shown in Figure 3. The minimum and maximum errors were
$-3.84 \mathrm{~m}$ and $2.25 \mathrm{~m}$, respectively, and the root mean square error (RMSE) was $0.34 \mathrm{~m}$. The RMSE for the $90 \%$ of the evaluation points excluding the upper and lower errors was $0.26 \mathrm{~m}$. LE90, which means the absolute value error distance including $90 \%$ of the evaluation points, was $0.35 \mathrm{~m}$. Considering the spatial resolution of $4.02 \mathrm{~cm}$, these results indicate that there are many errors in the UAV DSM. In order to analyse the spatial distribution of the errors, the error map of the UAV DSM was created as shown in Figure 5.

As a result, we found that most of the large errors appeared on the left side of the target area. This is analyzed because the seaice surface of the left side is relatively more homogeneous, as shown in the ortho-mosaic and DSM of Figure 6. These results support our argument that it will be difficult to ensure high quality of DSM due to the characteristics of sea-ice.

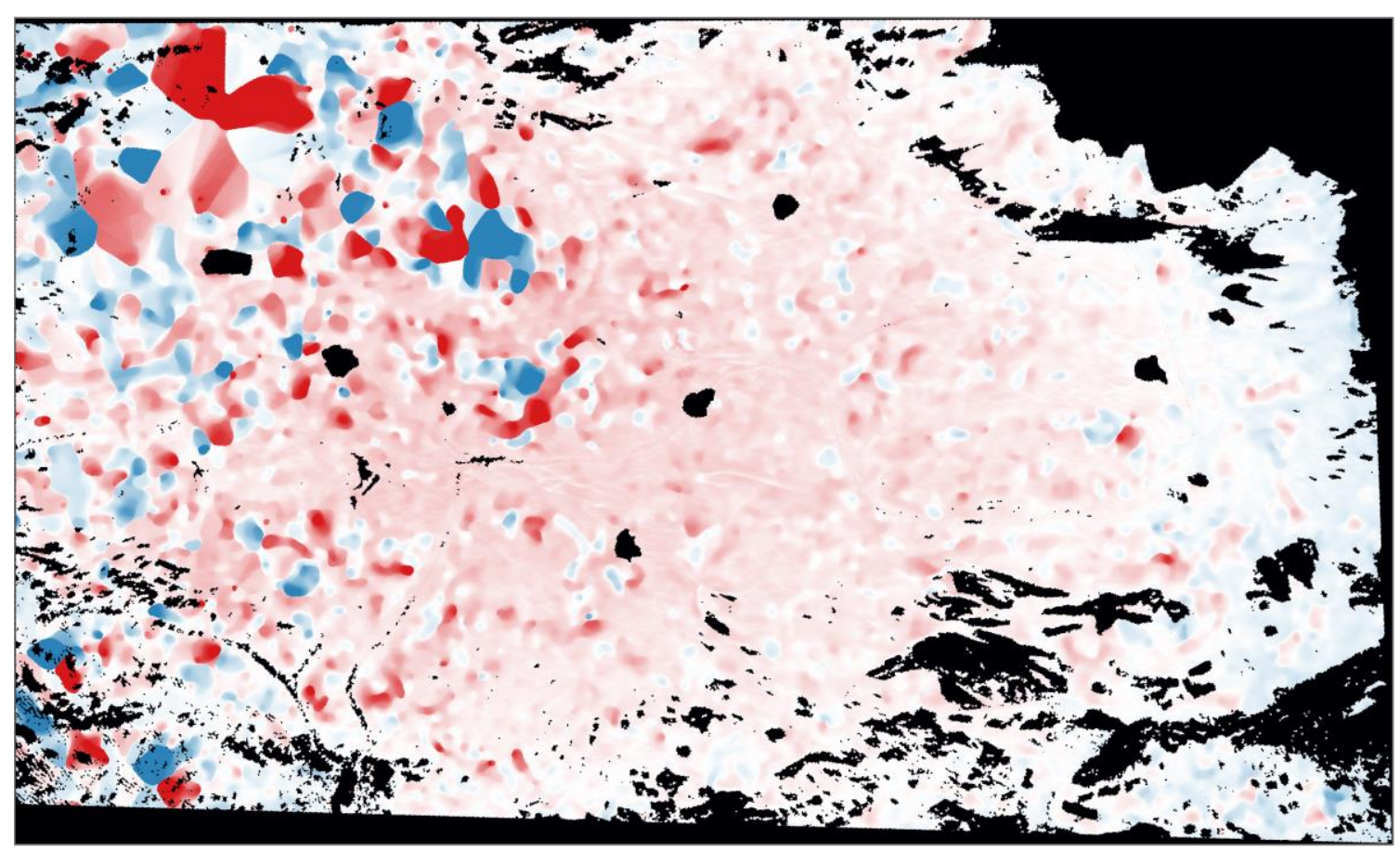

[m]

Figure 5. Error map for the DSM derived from UAV images. 


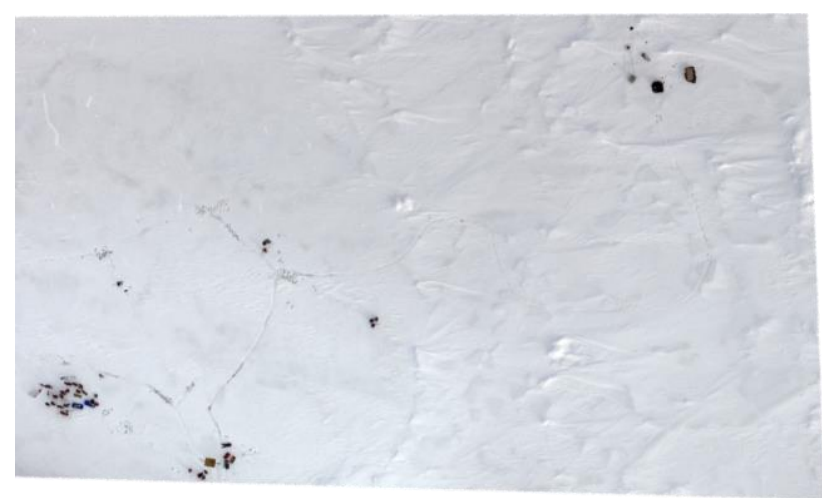

(a)

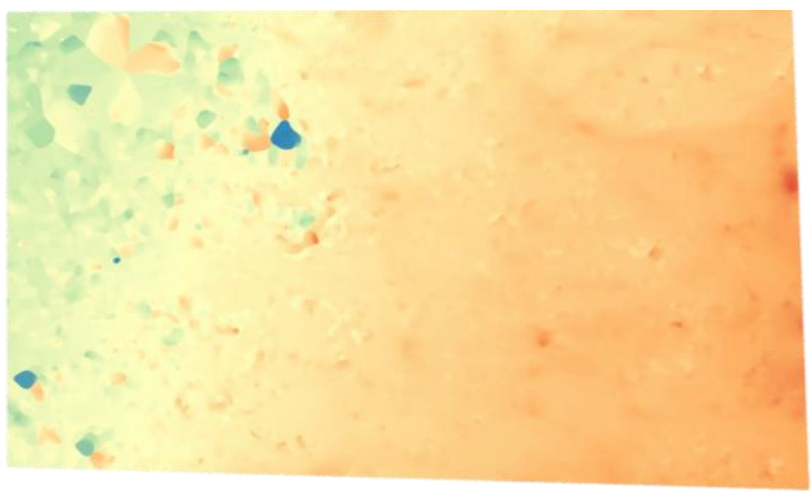

(b)

Figure 6. Ortho-mosaic and DSM derived from UAV images for the target area. (a) ortho-mosaic and (b) DSM.

Incorrect match detection was carried out for the target area of the UAV DSM. Range of search window sizes was set from $7 \times 7$ to $55 \times 55$. Zero mean normalized cross-correlation (ZNCC) was used as a similarity measure. ZNCC $0.3,0.4$, and 0.5 were applied as the matching success criterion, respectively. Figure 7 shows detection results for the target area.

The experimental results show that the proposed method can effectively detect errors in the UAV DSM. As the matching success criterion was increased, the areas of the detected errors were also enlarged. When the criterion was larger than ZNCC 0.5, we confirmed that the detection result could faithfully reflect the errors in the DSM.

For quantitative evaluation, we compared the error map of UAV DSM with the detection results. In this experiment, only matches that have an error greater than $0.2 \mathrm{~m}$ were regarded as incorrect matches. Detection accuracy was the highest when ZNCC 0.5 was used as the criterion, as in the visual analysis. The accuracy was $49 \%, 64 \%$, and $76 \%$ for ZNCC $0.3,0.4$, and 0.5 , respectively Figure 8 shows the comparison result between the error map and the detection result with the criterion of ZNCC 0.5.

If a higher criterion is applied, more errors would be detected. If so, however, the number of incorrect matches to be corrected later will also increase. Therefore, the matching success criterion should be determined by taking into account the trade-off between accuracy requirement and computational efficiency depending on the purpose of application.
Error Detection Results

[m]
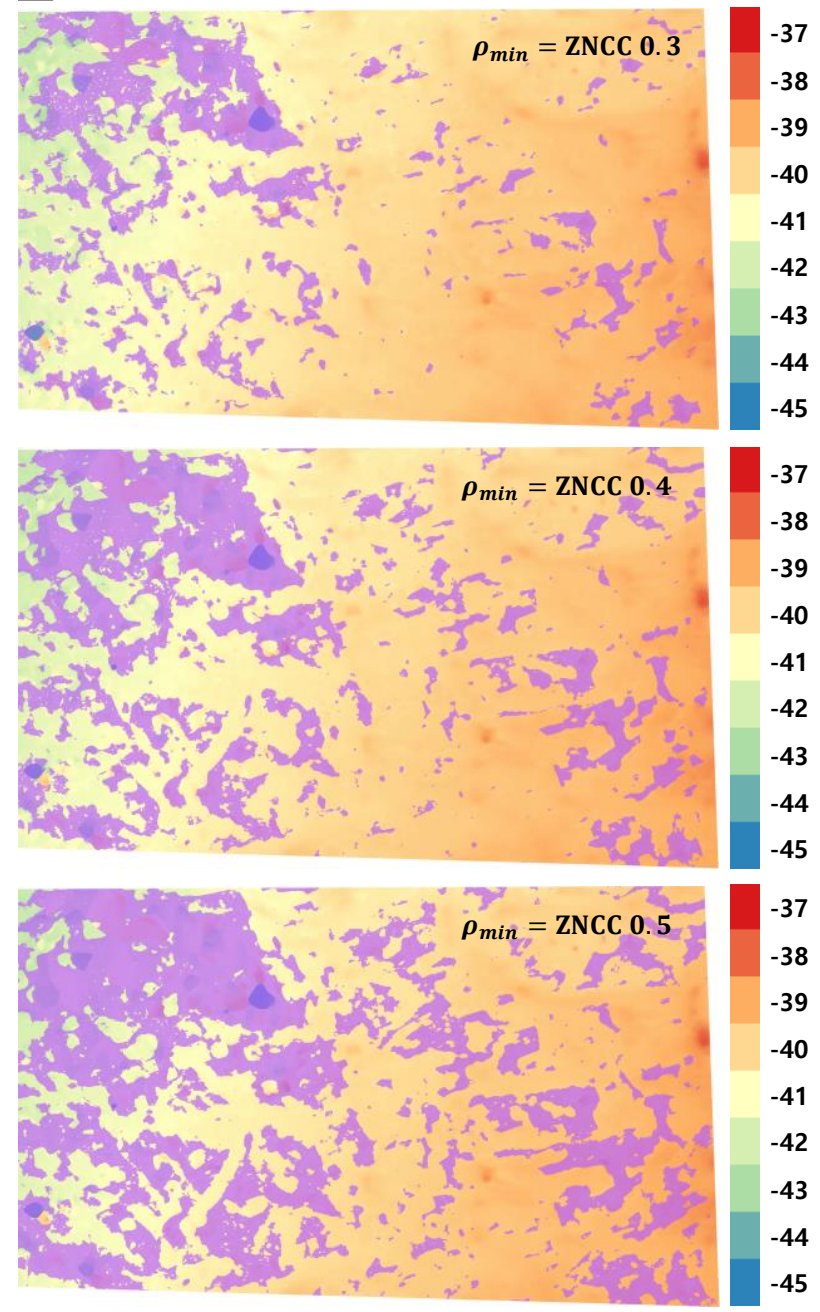

Figure 7. Incorrect match detection results for the target area.

\section{CONCLUSION}

In this paper, we have studied a method for effectively detecting incorrect matches in a sea-ice DSM derived from UAV images. The proposed method makes it possible to adjust search window size to identify incorrect matches due to characteristic of textureless surface.

In order to analyze the performance of the proposed method, we generated a DSM using UAV images taken at sea-ice camp, and then registered with laser scanning data acquired for the same target area. Experimental results showed that the matching error of the DSM was distributed along textureless surfaces and that the proposed method reliably detected these errors.

The proposed method is meaningful in that it can not only quantify the performance of sea-ice DSMs derived from UAV images without GCPs, but also contribute to improve efficiency of DSM correction to be performed later. In future work, we will investigate ways to enhance the performance of the proposed method, and then, based on the proposed method, develop an effective correction method that can improve the quality of seaice DSMs. 


\section{Error Detection Results}

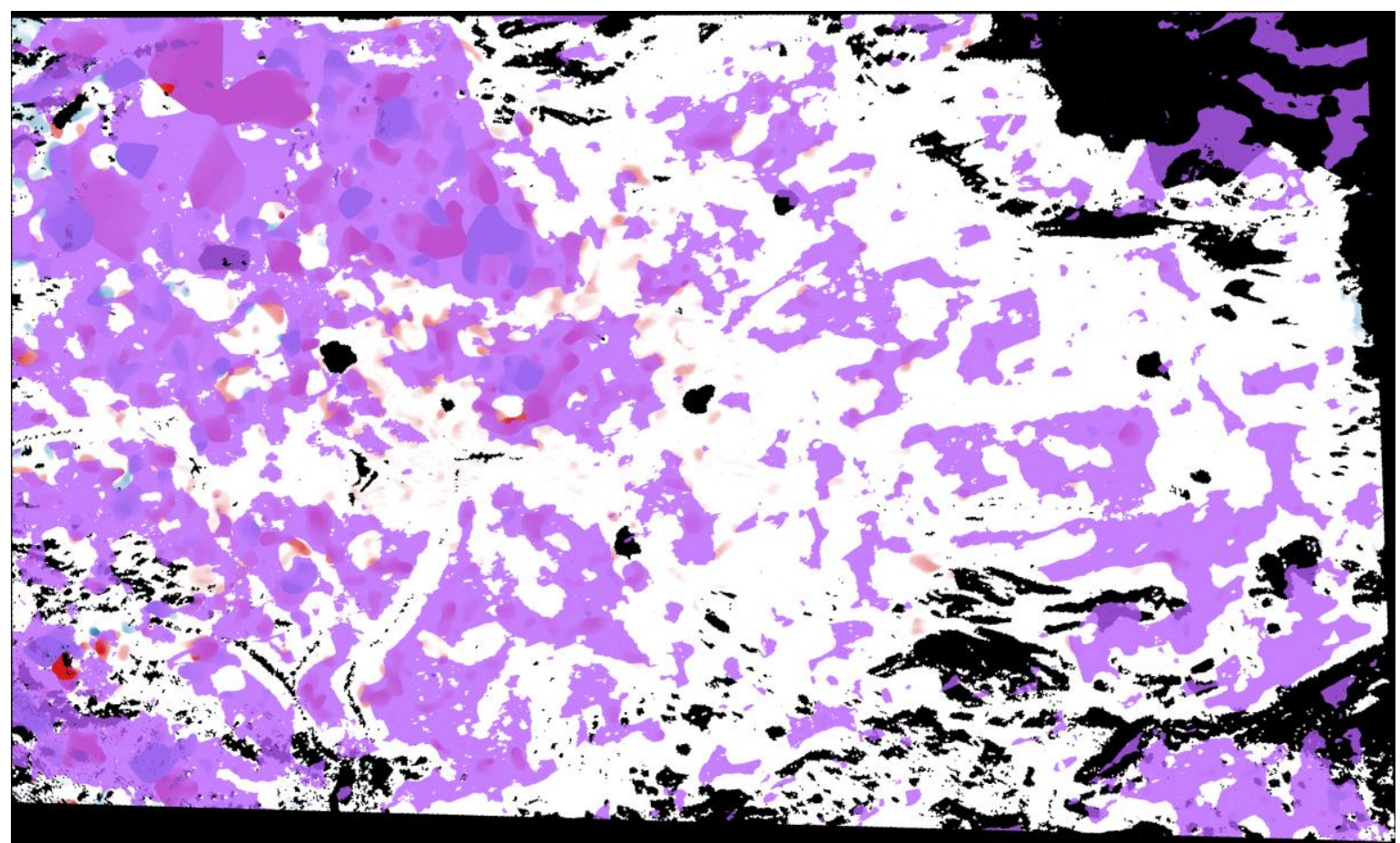

Figure 8. Comparison result between the error map and the detection result with the criterion of ZNCC 0.5. [m]

1.0

0.9

0.8

0.7

0.6

0.5

0.4

0.3

0.2

0.1

0

$-0.1$

$-0.4$

$-0.5$

$-0.6$

$-0.7$

$-0.8$

$-0.9$

$-1.0$

\section{REFERENCES}

Divine, D.V., Pedersen, C.A., Karlsen, T.I., Aas, H.F., Granskog, M.A., Hudson, S.R., and Gerland, S., 2016. Photogrammetric retrieval and analysis of small scale sea ice topography during summer melt. Cold. Reg. Sci. Technol. 129, pp.77-84.

Hagen R., Peters M., Liang R., Ball D., and Brozena J., 2014. Measuring Arctic sea ice motion in real time with photogrammetry. IEEE Geosci. Remote Sens. Lett., 11(2014), pp. 1956-1960.

Tschudi, M. A., Maslanik, J. A., and Perovich, D. K., 2008. Derivation of melt pond coverage on Arctic sea ice using MODIS observations. Remote Sens. Environ., 112, pp.2605-2614.

Veksler, O., 2003. Fast Variable Window for Stereo Correspondence using Integral Images, Proc. IEEE Conf. Computer Vision and Pattern Recognition, vol. 1, pp. 556-561. 